\title{
DISTRIBUTION OF RELATIVE PLATE MOTION ALONG THE PACIFIC-NORTH AMERICAN PLATE BOUNDARY DETERMINED FROM MOBILE VLBI MEASUREMENTS
}

\author{
P. M. Kroger, G. A. Lyzenga, K. S. Wallace, and J. M. Davidson \\ Jet Propulsion Laboratory, California Institute of Technology \\ 4800 Oak Grove Drive, MS 238-640 \\ Pasadena, California 91109
}

\section{INTRODUCTION}

The problem of understanding the deformation occurring along the Pacific-North American plate boundary in the western United States depends upon understanding the forces which drive the plates in this region. One of the primary sources of our knowledge concerning these forces lies in their manifestation as relative displacements which occur throughout the broad zone of deformation surrounding the San Andreas fault system. It is information concerning the spatial and temporal distribution of these motions which will be of greatest benefit in helping to determine which of several possible mechanisms is responsible for driving contemporary plate motions in this region.

The resolution of the motions in this region is itself proving to be a problem of considerable magnitude. One of the principal questions involving the present day motions is the apparent discrepancy between the relative plate rate of $5.6 \mathrm{~cm} / \mathrm{yr}$ predicted by rigid plate models and the slip rates across the San Andreas fault of 2.5-3.5 cm/yr which have been determined from geologic and earth-based geodetic measurements. There is a strong possibility that this "missing motion" is occurring west of the San Andreas fault, probably in association with offshore faults. Measurements using the Global Positioning System (GPS) satellites have recently extended the coverage of geodetic observations to island sites off the southern California coast, but it will require at least several years of such measurements before accurate velocities to these offshore sites can be inferred.

\section{RESULTS}

We have used over 6 years of mobile VLBI baseline measurements in this region to determine the velocities of 11 sites relative to North America (see Figure 1.). We find these velocities to be generally consistent with those determined from geologic data and contemporaneous satellite laser ranging measurements made in the same region.

The most striking feature of the results presented in Figure 1 is the contrast between the local distribution of velocities in northern and southern California. In southern California the velocities of sites west of the San Andreas fault increase gradually with increasing distance west of the fault reaching a maximum value of $4.1 \mathrm{~cm} / \mathrm{yr}$ at Vandenberg AFB on the Pacific coast approximately $100 \mathrm{~km}$ west of the fault. In northern 
California, on the other hand, velocities of 4.6 and $4.5 \mathrm{~cm} / \mathrm{yr}$ are obtained for the sites at Ft. Ord and $\mathrm{Pt}$. Reyes located within $15 \mathrm{~km}$ of the fault. It is noteworthy that even the westernmost of the VLBI sites in southern California falls significantly short of the full $5.6 \mathrm{~cm} / \mathrm{yr}$ predicted by rigid plate models.

\section{DISCUSSION}

We find that once transient cyclic deformation associated with the earthquake cycle is taken into account, the observed geodetic site motions are consistent with the activity of known subsidiary faults to the San Andreas and with the $5.6 \mathrm{~cm} / \mathrm{yr}$ plate motion rate.

These conclusions are reached through consideration of a finite element model of elastic deformation in the lithosphere. The aim of the modeling is to attempt an explanation of the apparent variations in strain distribution along the strike of the San Andreas system. In particular, a suite of two-dimensional models of time dependent response in the coupled lithosphere-asthenosphere system is considered. These models provide synthetic displacement profiles across three typical cross sections of the plate boundary.

The three "slices" perpendicular to the faults are differentiated by the number and location of active faults with respect to the plate boundary. While the results of these calculations are successful in reproducing both classical geodetic and VLBI results, limitations in the coverage and precision of the available data preclude the placing of strong constraints upon the parameters of these theoretical models. This fact points out the need for a much denser spatial network of observing sites if more sophisticated models of interplate deformation in this region are to be successfully tested.

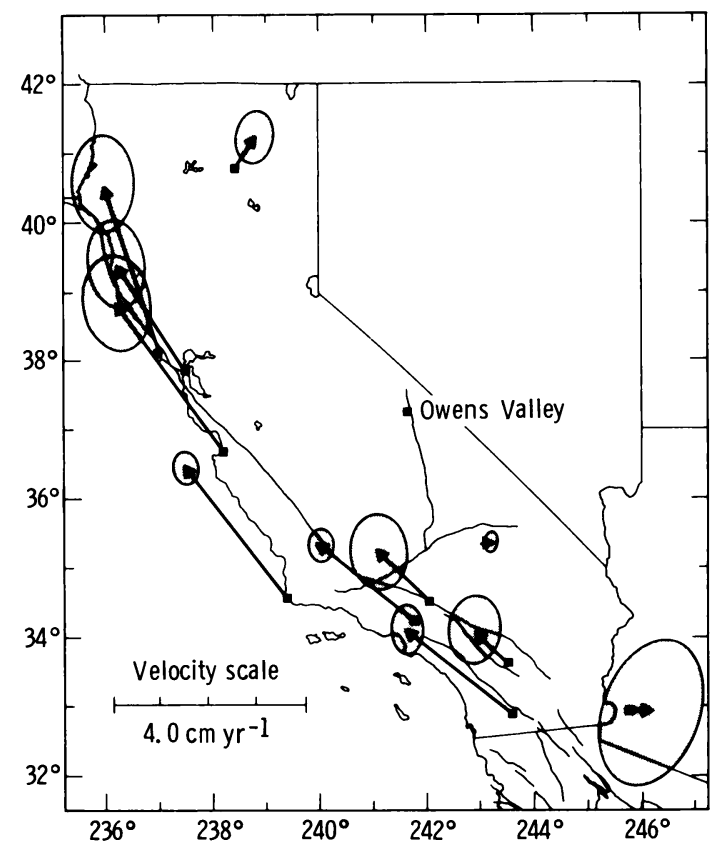

Figure 1. Velocities of VLBI sites relative to the site at the Owens Valley Radio Observatory which is assumed fixed. Error ellipses represent one standard deviation. 